\title{
Novas utilizações da cetamina para tratamento da dor somática e seus mecanismos de ação
}

\author{
[New uses for ketamine in the treatment of somatic pain and its action mechanisms]
}

\section{"Revisão/Review"}

\author{
Marco Aurélio Camargo Fontanela ${ }^{1}$, Marilda Onghero Taffarel ${ }^{1 *}$, \\ Carlos Rodrigo Komatsu de Alencar ${ }^{2}$
}

\begin{abstract}
${ }^{1}$ Curso de Medicina Veterinária, Universidade Estadual de Maringá, campus Regional de Umuarama-PR, Brasil. ${ }^{2}$ Programa de Pós-Graduação em Produção Sustentável e Saúde Animal, Universidade Estadual de Maringá, Campus Regional de Umuarama-PR, Brasil.

*Autor para correspondência/Corresponding author: E-mail: motaffarel@uem.br
\end{abstract}

\begin{abstract}
Resumo
A cetamina é classificada como anestésico dissociativo, seu emprego caiu em desuso graças a formulação de novos fármacos com menor apresentação de efeitos adversos. No entanto, estudos recentes têm apontado para qualidades até então desconhecidas da cetamina. $\mathrm{O}$ objetivo dessa revisão foi relatar os efeitos farmacológicos antálgicos periféricos e centrais da cetamina, bem como sua possível aplicabilidade clínica para o tratamento da dor somática. Vários estudos vêm apontando para uma função antiinflamatória, também como adjuvante de anestésicos locais, ou ainda que baixas doses para propriedades analgésicas apenas, sem observação de efeitos adversos e via de administração intranasal como uma opção não invasiva de administração. Assim, a cetamia tem se tornado uma excelente alternativa para os protocolos multimodais de tratamento antinociceptivo, e não somente um anestésico dissociativo de baixo custo e muitos efeitos adversos. Conclui-se que a cetamina possui diferentes mecanismos de ação dos já elucidados, e que sua aplicabilidade clínica pode ser diferencial quando utilizada em um protocolo analgésico multimodal, melhorando a qualidade de tratamento aos pacientes.
\end{abstract}

Palavras-chave: analgesia; anti-inflamatório; anestésico.

\begin{abstract}
Ketamine is classified as a dissociative anesthetic; it has fallen into disuse due to the formulation of new drugs with less adverse effects. However, recent studies have pointed to previously unknown qualities of ketamine. The objective of this review was to report the peripheral and central pharmacological analgesic effects of ketamine, as well as its possible clinical applicability for the treatment of somatic pain. Several studies have pointed to an anti-inflammatory function, also as an adjunct to local anesthetics, or even low doses for analgesic properties alone, without observing adverse effects, and via intranasal administration as a noninvasive administration option. Thus, ketamine has become an excellent alternative for multimodal antinociceptive treatment protocols, and not only a low cost dissociative anesthetic with many adverse effects. It is concluded that ketamine has different mechanisms of action than those already elucidated, and that its clinical applicability can be a differential when used in a multimodal analgesic protocol, improving the quality of treatment to patients.
\end{abstract}

Keywords: analgesia; anti-inflammatory; anesthetic.

\section{Introdução}

Os anestésicos dissociativos compõem uma categoria de fármacos amplamente utilizada em medicina veterinária e em seres humanos (Romagnoli et al., 2017). A cetamina é um derivado da fenciclidina, e quimicamente é

designada como 2-(Oclorofenil)-2-(Metil-amino)ciclo-hexanona. A forma comercialmente utilizada é composta de uma mistura de seus isômeros $(+)$ e (-). Os isômeros ópticos têm fórmula estrutural e química muito semelhante, sendo diferenciadas 
apenas no arranjo do átomo de carbono quiral. Embora as propriedades físicas sejam idênticas, apresentam a propriedade de desviar a luz para lados opostos e ocupam posições diferentes no espaço, diferindo em propriedades farmacodinâmicas e farmacocinéticas. O Isômero (+) é descrito como sendo três vezes mais potente como anestésico, e duas a quatro vezes mais potente como analgésico, quando administrado pela via sistêmica. É hidrossolúvel, e uma solução aquosa a $10 \%$ tem $\mathrm{pH}$ de 3,5 . Sua lipossolubilidade é de aproximadamente dez vezes a do tiopental, sendo rapidamente absorvida após sua administração. A biotransformação da cetamina é complexa e envolve metabolismo oxidativo em vários locais no anel ciclo-hexanona, bem como Ndesmetilação, formando vários metabólitos, entre eles a norcetamina, que é um metabolito quantitativamente importante, já que o mesmo possui potência anestésica de aproximadamente um $1 / 5$ a $1 / 3$ da cetamina, prolongando assim seus efeitos anestésicos (Fantoni et al., 2011).

A cetamina é denominada como anestésico dissociativo por ser um fármaco capaz de interromper a transmissão ascendente, em regiões centrais responsáveis por funções conscientes e inconscientes, não deprimindo de maneira generalizada todos os centros cerebrais (Corssen, 1968). Seu mecanismo de ação é descrito como depressão seletiva da atividade neuronal no eixo neocorticotalâmico e no núcleo central do tálamo coincidente envolvendo algumas estruturas do sistema límbico, como o hipocampo (Miyasaka e Domino, 1968; Massopust et al., 1973).

Apesar da condecoração da cetamina como anestésico dissociativo, suas características antálgicas possibilitaram um reconhecimento maior desse fármaco como analgésico, quando o mesmo é utilizado em doses baixas ou doses subanestésicas. Sendo que o efeito analgésico destas é tão eficaz quanto das doses anestésicas, porém sem apresentar os efeitos adversos (RascónMartínez et al., 2016; Allen e Ivester, 2017; Motov et al, 2017; Parvizrad et al., 2017; Pourmand et al., 2017). O efeito analgésico é proveniente do antagonismo não competitivo pelos receptores do tipo N-metil-D-aspartato (NMDA), importantes sítios de ligação para anestésicos presentes no sistema nervoso central, sendo o glutamato o principal neurotransmissor excitatório que age nestes receptores. Também já se é conhecido o seu papel agonista opioide (receptores $\mu, \delta, \kappa$ ), do sistema monoaminérgico inibidor descendente, e dos receptores muscarínicos (Luft e Mendes, 2005; Muir, 2010; Berry, 2017). Outras ações deste fármaco incluem a ação nos canais de sódio, potássio e cálcio; depressão dos receptores de acetilcolina; aumento e prolongamento da ligação do cálcio ao ácido gama-aminobutírico (GABA); depressão das células nociceptivas da formação reticular bulbar medial e da ativação das células localizadas nas lâminas I e V do corno medular dorsal (Brockmeyer e Kendig, 1995; Ohtani et al, 1979; Kitahana et al, 1973). Também existem outras características farmacológicas da cetamina como sua capacidade em inibir a liberação de fator de necrose tumoral (TNF-alfa), interleucina-6 e citoquinas (Liu et al., 2012; Preskorn, 2012; Robson et al., 2012; Farmawy e Rashad, 2014). Em equinos essa peculiaridade de mecanismos da cetamina parece ser de extrema relevância para o tratamento da dor, uma vez que outros antagonistas de receptores NMDA não apresentam qualidade antálgica (Muir, 2010).

Com base nas propriedades analgésicas da cetamina, objetiva-se com esta revisão de literatura relatar os efeitos farmacológicos antálgicos periféricos e centrais da cetamina, bem como sua possível aplicabilidade clínica para o tratamento da dor somática.

\section{Desenvolvimento}

A analgesia promovida pela cetamina se dá principalmente na dor de origem somática (Lin, 2013). Acredita-se que o emprego da mesma é mais útil para as anestesias e analgesias pós-operatórias que envolvam intervenções cirúrgicas tegumentares e musculoesqueléticas superficiais (Sawyer et al., 1990; Sawyer et al., 1993). Contudo, estudos demonstraram que a administração local de cetamina pode produzir um curto efeito anestésico local (Warncke et al., 1997; Pederson et al., 1998), esses mecanismos de ação estão sendo elucidados com novos estudos e serão discutidos no decorrer do trabalho.

A supressão da inflamação tem sido um novo campo de estudo e utilização da cetamina. Dale et al. (2012) realizaram um estudo de metaanálise cujos resultados demonstraram que a cetamina no trans-operatório diminuiu o processo inflamatório imediato. Segundo Bajwa (2017), esse efeito ocorre pela inibição da atividade dos linfócitos, células "natural killer" e neutrófilos. Além da supressão da atividade tática da quimioterapia e a produção de oxidantes por neutrófilos. Tanto o TNF-alfa como o óxido nítrico 
desempenham um papel importante durante a inflamação, e a inibição destas atividades pode afetar a imunidade mediada pelos macrófagos. Evidências in vitro apoiam essa teoria de como a cetamina exerce efeitos supressores sobre a função dos macrófagos, uma vez que a cetamina diminui o estresse oxidativo e resposta de citocinas. Além de reduzir a produção de TNF-alfa e óxido nítrico em macrófagos (Tvwrkoy et al, 1996; Xingwei et al, 2014). Kocket al. (2013) citaram também como mecanismos anti-inflamatórios a inibição da proteína-1 do ativador da transcrição e o fator nuclear (NF), juntamente com a diminuição dos níveis de proteína $\mathrm{C}$ reativa. Ademais RascónMartinez et al. (2016) citam em seu trabalho que doses baixas de cetamina em humanos $(0,5 \mathrm{mg} / \mathrm{kg})$ é capaz de diminuir os níveis séricos de proteína $\mathrm{C}$ reativa com uma única aplicação.

Esse efeito anti-inflamatório da cetamina parece ser benéfico para uso desse fármaco em casos de sepse, uma vez que a resposta inflamatória exerce importante papel na fisiopatologia dessa afecção, causando imunossupressão e contribuindo para a alta mortalidade. No entanto se faz necessária a utilização no início do processo para efetividade da terapia, e ainda a necessidade de novos estudos para melhor elucidar o mecanismo de ação (Tsao e Wu 2012). Os processos de endotoxemia estimulam fagócitos mononucleares a produzir e liberar mediadores inflamatórios. Essa patogênese é de extrema importância em equinos com este quadro, como é o caso da laminite, pois até mesmo em doses baixas administradas de cetamina foi capaz de suprimir a resposta inflamatória sistêmica e diminuir a expressão de proteínas derivadas do processo endotóxico (Lankveld et al, 2005; Rascón-Martinez et al, 2016). Já em animais saudáveis a utilização de infusão de cetamina não surtiu efeitos quando realizados testes álgicos sobre o casco de equinos, o que salienta ainda mais a importância da mesma em relativo aos efeitos supressores de inflamação e seus benefícios (Fielding et al., 2006).

Danos aos tecidos, anestesia, dor pósoperatória e estresse psicológico afetam o sistema imunológico do paciente no período perioperatório. $\mathrm{O}$ trauma cirúrgico pode induzir um complexo sistema de cascatas de citocinas com diferentes efeitos sobre o paciente, uma vez que certas citocinas pró-inflamatórias são excessivamente estimuladas e a imunidade mediada por células é suprimida. Esta estimulação excessiva de prócitocinas inflamatórias resultam em hipotensão, choque e falência de múltiplos órgãos. Assim, é importante o tratamento da dor pós-operatória de modo a reduzir a supressão da resposta imune mediada por linfócitos e atenuar as reações próinflamatórias das citocinas à cirurgia. Dessa forma, a cetamina exerce papel anti-inflamatório importante, principalmente ao reduzir os níveis séricos de IL-6 no pós-operatório (Liu et al., 2012).

No entanto, em estudo em humanos, duplocego, realizado por D'Alonso et al. (2011), obtevese como resultado que a utilização da cetamina no pré-operatório de cirurgia torácica não apresentou redução dos níveis de IL-6 nos pacientes. De outra forma, os mediadores já citados, provocam estimulação nociceptiva contínua das fibras $\mathrm{C}$, que por sua vez ativa os receptores NMDA no sistema nervoso central (SNC). A ativação desses receptores diminui o limiar de ação do glutamato nas fibras $\mathrm{C}$, tornando-as assim mais sensíveis aos estímulos nociceptivos. Isso resulta em uma amplificação da resposta aos estímulos, que se caracteriza por maior sensibilização após dor primária, o que pode piorar o quadro pósoperatório do paciente (Woolfe e Thompson, 1991; Stubburg et al., 1997).

A fim de minimizar este fenômeno, o uso de infusões contínuas de doses sub-anestésicas de cetamina, associada ou não a outros analgésicos, tem sido utilizada a fim de minimizar a esta sensibilização central (Fu et al., 1997; Kwok et al., 2004). Laskowski et al. (2011) concluíram que esse efeito analgésico independe do tipo de opioide intra-operatório administrado, tempo de administração da cetamina e dose de mesma. Kawamata et al. (2000) estudaram os mecanismos de ação da cetamina para inibição da dor e da hiperalgesia, concluindo que, quanto ao tratamento da dor, a cetamina o promove através da ativação do sistema inibitório monoaminérgico; já o antagonismo NMDA é responsável pelo tratamento da hiperalgesia.

Além dos efeitos anti-inflamatórios citados, alguns estudos apontaram para um sinergismo da cetamina e anestésicos locais, demonstrando que a associação da cetamina à bupivacaína aumenta a duração do bloqueio anestésico, até duplicando o mesmo. Esse efeito de analgesia periférica do fármaco pode ser atribuído ao bloqueio das correntes de sódio e potássio dos nervos periféricos, bloqueio dos receptores NMDA, e bloqueio dos receptores de ácido alfa-amino-3hidróxi-5-metil-4-isoxazolepropiõnico (AMPA) (Tvwrkoy et al., 1996; Brau et al., 1997; Lopez- 
Sanrom et al., 2003;). Stoetzer et al. (2017) também refiram que a associação da bupivacaína à cetamina resultou em potencialização do bloqueio dose dependente, resultante do bloqueio tônico dos canais de sódio. Sendo que Moharari et al. (2016) ainda complementaram em seu estudo que o uso dessa associação foi capaz, não só de, potencializar os efeitos analgésicos, mas também aumentar o tempo para resgate antálgico pós-cirúrgico e a dose necessária para o mesmo quando comparada com o uso isolado de bupivacaína ou cetamina quando intravenosa. Também Othman et al. (2016) concluíram que foi necessário menor uso de opioides para resgate em pacientes que receberam bupivacaína mais cetamina, em bloqueio para cirurgia de mastectomia em humanos, quando comparado com apenas o anestésico local isolado. Contudo, Bailard, et al. (2014) não recomendaram a utilização de cetamina como adjuvante para a bupivacaína rotineiramente devido à escassez de dados de suporte, baixa eficácia e principalmente devido aos efeitos adversos que a mesma pode apresentar.

Por outro lado, Farmawy e Rashad (2014) compararam o bloqueio local da cetamina, quando comparado à ropivacaína, onde foi demonstrado um efeito analgésico superior da última em relação ao grupo cetamina, no entanto os autores avaliaram esses fármacos apenas de maneira isolada. Diferente do trabalho de Hong et al. (2017), que a associação da cetamina pós-tonsilectomia em crianças foi capaz de potencializar os efeitos anestésicos da ropivacaína na dor pós-operatória imediata. Os autores observaram que o uso isolado da ropivacaína resultou em necessidade de resgate analgésico uma hora após o procedimento cirúrgico. Contudo, os pacientes que receberam ropivacaína associada à cetamina necessitaram de resgate analgésico, em média, 24 horas após o procedimento. Além disso, no grupo que recebeu associação não houve sinergismo em seus efeitos adversos. Porém, Lee et al. (2002) sugeriram em sua pesquisa que o uso combinado de cetamina mais ropivacaína não apresentou aumento dos efeitos de bloqueio e ainda que a presença de efeitos adversos é mais pronunciada nesses casos.

Quando utilizada por via epidural a cetamina foi capaz de induzir uma analgesia somática profunda, com menos efeito analgésico visceral (El-Khateeb, 1990; Guinto-Enriquez et al., 1990). Utilizada da mesma forma em equinos, a cetamina fornece uma analgesia adequada para realização de procedimentos perineais, onde também pode ser observado efeito sedativo (Gomes De Segura et al., 1998; Rédua et al., 2002). Também em equinos, Lopez-Sanroman et al (2003) utilizaram a cetamina para a realização de bloqueio de sesamoide abaxial e concluiram que a mesma é capaz de produzir adequada analgesia nessa situação, com início de ação de 2 minutos e tempo máximo de duração de 15 minutos. DeRossi et al. (2011) determinaram os efeitos analgésicos locais e sistêmicos da associação de cetamina com anestésicos locais em cães para bloqueio peridural torácico por punção lombosacra. Os animais foram distribuídos em três grupos recebendo administração peridural de lidocaína, cetamina ou associação de ambas. Os autores observaram que os animais que receberam a associação de lidocaína e cetamina, diferente dos outros grupos, apresentaram sedação leve e redução da pressão arterial sistólica e média. Já o tempo de analgesia da associação foi mais que o dobro dos outros protocolos estudados. DeRossi et al. (2009) também estudaram os efeitos da mesma associação administrada pela via peridural em gatos sedados com xilazina. Os resultados obtidos demonstraram que o protocolo foi capaz e induzir analgesia prolongada que se estendeu do coccígeo até a junção toraco-lombar. Boettger et al. (2010) compararam a utilização de cetamina e morfina por via epidural para o tratamento de artrite crônica induzida em ratos. Nesse estudo constatou-se que a cetamina apresentou melhor controle da dor, enquanto que o grupo que recebeu morfina apresentou maior redução da resposta inflamatória, o que foi observado por meio de análise histopatológica.

Efeitos de uso tópico da cetamina associada com outros anestésicos têm sido estudados e os achados também evidenciaram que esse tipo de protocolo apresentou melhor controle da dor. Moharari et al. (2010) compararam o uso de gel de lidocaína isolado ou somado à cetamina em humanos para cirurgia de cistoscopia e concluíram que incorporar a cetamina no gel tornou o procedimento mais suportável. Comparada com a lidocaína em apresentação de spray local, a cetamina também nessa apresentação demonstrou melhor analgesia após 40 minutos da cirurgia de tonsilectomia em crianças, enquanto a lidocaína apresentou-se melhor analgésico apenas antes desses 40 minutos (Hosseini Jahromi et al., 2012), o que segundo o autor se deve pelas características anti-inflamatórias da cetamina.

Também é possível de se encontrar na literatura o uso de local de cetamina associada ao 
tramadol. Em seu estudo, Khajavi et al. (2016) concluíram que a infiltração subcutânea combinada de cetamina e tramadol no local da incisão, para realização de pielolitotomia, produziu melhor analgesia e um efeito poupador de opioides durante as primeiras 24 horas, quando comparado com o grupo controle e os grupos que receberam uma infiltração subcutânea de apenas cetamina ou tramadol. Além disso, Ayoglu et al. (2010), em estudo realizado com humanos, demonstraram que a combinação de cetamina mais tramadol teve mais eficiência analgésica que o uso de ropivacaína ou tramadol isolados, ou a combinação cetamina com ropivacaína, para o uso intra-articular. Nesse caso é importante salientar que os opioides também possuem capacidade analgésica locais em baixas doses sobre tecidos inflamados, sendo amplamente utilizados em artroscopias. (Luna et al., 2016). Todavia Rosseland et al. (2003) concluíram em seus ensaios que isoladamente a cetamina pela via intra-articular obteve a mesma capacidade analgésica que a solução salina, além disso, que a cetamina foi mais eficiente para o tratamento da dor quando optada pela administração intramuscular comparada com a via intra-articular.

No entanto, também há relatos que devido seu baixo $\mathrm{pH}$, a cetamina seja irritante para os tecidos, e os animais apresentam sinais de dor quando aplicado via intramuscular (IM), porém não apresentando edema ou necrose tecidual (Fantoni et al., 2011). Uma alternativa para prevenção dessa adversidade é a alcalinização da cetamina. Além disso, Lopez-Sanroman et al (2003), concluiram em seu estudo que realizar esse procedimento aumenta a capacidade analgésica local da cetamina.

Outra via de administração desse fármaco é a via para intranasal. Estudos têm demonstrado ser uma alternativa de rápida absorção, não invasiva e não dolorosa. Essa via apresenta boa absorção e foi investigada para a aplicabilidade em vários tipos de dor, como por exemplo, pacientes com câncer e dor aguda após trauma, ou no pós-operatório (Christensen et al., 2017). Em gatos, Marjani et al. (2015) concluiram que a administração de cetamina mais midazolan intranasal foi capaz de causar sedação, com pico de ação mais rápido quando comparado com realização de aplicação intramuscular. No entanto, neste estudo não foi investigado a capacidade antálgica desse protocolo. Em humanos, quando comparada com a utilização de Morfina pela via intravenosa, para alívio da dor em cólicas renais, ou extração de molar, a cetamina intranasal se mostrou estatisticamente com a mesma eficácia (Liao et al., 2008; Farnia et al., 2016). Além de que, a adição de cetamina com a morfina pela via intravenosa para tratamento de cólica renal em humanos demostrou um controle da dor excelente (Abbasi et al., 2017). Já quando comparado com a aplicação de fentanil, também intranasal, a cetamina obteve menor controle analgésico para cirurgia nasal endoscópica em humanos, além de manter seus efeitos adversos. Contudo, estes efeitos adversos ocorreram de maneira transitória e não necessitaram de intervenção (Abdel-Ghaffar e Salem, 2012). Já Andolfatto et al. (2013), também em humanos, referiam que o uso de morfina intravenosa ou fentanil intranasal podem resultar em depressão respiratória, a passo que a cetamina intranasal não apresentou nenhum efeito adverso grave, e foi capaz de oferecer controle da dor em $88 \%$ dos pacientes. Ademais, Parvizrad et al (2017) concluíram em seu estudo que a cetamina intranasal foi capaz de suprir um bom controle analgésico em pacientes humanos com traumas ortopédicos moderados à grave, além de apresentar poucos efeitos adversos.

\section{Considerações Finais}

A utilização de cetamina para o tratamento da dor somática parece possuir várias qualidades, sendo mediando à inflamação, ou através de bloqueio das fibras nervosas - potencializando assim os efeitos benéficos dos anestésicos locais , ou por sinergismo com opioides. Muitos mecanismos de ação da cetamina são pesquisados atualmente, no entanto ainda é necessário elucidar melhor a farmacocinética dos mesmos. A cetamina se mostra adequada para utilização clínica e cirúrgica para tratamento da dor, inclusive indicada em casos graves, como a sepse. No entanto, este fármaco deve ser utilizado com cautela devido aos seus efeitos adversos. Muitos profissionais fizeram desuso desse fármaco devido a esses efeitos e ao desenvolvimento de novas moléculas com melhor margem de segurança.

Contudo, o mesmo tem se mostrado seguro em estudos e com muitos benefícios, principalmente quando utilizado em associação, potencializando diversos fármacos, ou quando utilizado em baixas doses, causando assim alívio da dor e poucos efeitos adversos. Além da recente aplicabilidade pela via intranasal, que parece ser de qualidade parecida com a intravenosa e não é uma opção invasiva. 


\section{Conflito de interesse}

Os autores declaram não haver conflito de interesse.

\section{Referências}

Abbasi, S.; Bidi, N.; Mahshidfar, B.; Hafezimoghadam, P.; Rezai, M.; Mofidi, M.; Farsi, D. Canlow-dose of ketamine reduce the need for morphine in renal colic? A doubleblind randomized clinical trial. American Journal of Emergency Medicine, 36(6): 376379, 2017.

Abdel-Ghaffar, H.S.; Salem, M.A.M. Safety and analgesic efficacy of pre-empitive intranasal ketamine versus intranasal fentanyl in patients undergoing endescopic nasal surgery. Journal of American Science, 8(3): 430-436, 2012.

Allen, C.A.; Ivester, J.R. Ketamine for pain management - side effects \&amp; potential adverse events. Pain Management Nursing, 18(6): 372-377, 2017.

Andolfatto, G.; Willman, E.; Joo, D.; Miller, P.; Wong, W.; Koehn, M.; Dobson, R.; Angus, E.; Moadebi, S. Intranasal ketamine for analgesia in the emergencydepartment: a prospective observational series. Society for Academic Emergency Medicine, 20(10): 1050-1054, 2013.

Ayoglu, H.; Altunkaya A.H.; Bayar A.; Turan I.O.; Ozer, Y.; Ege, A. The effect of intra articular combinations of tramadol and ropivacaine with ketamine on postoperative pain after arthroscopic meniscectomy. Archives of Orthopaedic and Trauma Surgery, 130: 307312, 2010.

Bailard, N.S.; Ortiz, J.; Flores, R.A. Additives to local anesthetics for peripheral nerve blocks: Evidence, limitations, and recommendations. American Journal of Health-System Pharmacy, 71(5): 373-385, 2014.

Bajwa, S.J.S. Ketamine-the confluence of old and recent concepts. Journal of Pain \& Relief, 6: 283, 2017.

Berry, S.H., Anestésicos Injetáveis. In: Grimm, K. A.; Lamont, L. A.; Tranquilli, W. J.; Greene, S. A .; Robertson, S. A., Lumb \& Jones Anestesiologia e Analgesia em Veterinária, Rio de Janeiro: Guanabara Koogan, 2017, p. 271-290.

Boettger, M.K.; Weber, K.; Gajda, M.; Bräuer, R.; Schaible, H. Spinally applied ketamine or morphine attenuate peripheral inflammation and hyperalgesia in acute and chronic phases of experimental arthritis. Brain, Behavior, and Immunity, 24(3): 474-485, 2010.

Brau, M.E.; Sander, F.; Vargel, W.; Hemelmann, G. Blocking mechanisms of ketamine and its enantiomers in enzymatically demyelinated peripheral nerve as revealed by single-channel experiments. Anesthesiology, 86(2): 903, 1997.

Brockmeyer, D.M.; Kengid, J.J. Seletective effects of ketamine on amino acid-mediated pathways in neonatal rat spinal cord. British Journal of Anaesthesia, 74(1): 79, 1995.

Corssen, G.; Miyasaka, M.; Domino, E.F., Changing concepts in pain control during surgery: Dissociative anesthesia with CI-581-A progress report. Anestheanlg, 47(6): 746, 1968.

Christensen, K.; Rogers, E.; Green, G.A.; Hamilton, D.A.; Mermelsteins, F.; Liao, E.; Writght, C.; Carr, D.B. Safety and efficacy of intranasal ketamine for acute postoperative pain. Acute Pain Elsevier, 9(4): 183-192, 2007.

Dale, O.; Somogyi, A.A.; Li, Y.; Sullivan, T.; Shavit,Y. Does intraoperative ketamine attenuate inflammatory reactivity following surgery? A systematic review and metaanalysis. Pain Medicine, 115(4), 2012.

D'Alonso, R.C.; Bennett-Guerrero, E.; Podgoreanu, M.; D'Amico, T.A.; Harpole, D.H.; Shaw, A.D. A randomized, double blind, placebo controlled clinical trial of the preoperative use of ketamine for reducing inflammation and pain after thoracic surgery. Journal of Anesthesia, 25(5): 672-678, 2011.

DeRossi, R.; Benites, A.P.; Ferreira, J.Z.; Neto, J.M.; Hermeto, L.C. Efeitos da cetamina e lidocaína peridural lombossacra em gatos sedados com xilazina. Journal of the South African Veterinary Association, 80(2): 79-83, 2009.

DeRossi, R.; Frazílio, F.O.; Jardim, P.H.; Martins, A.R.; Schimidt, R.; Negrini-Net, J.M. Evaluation of thoracic epidural analgesia induced by lidocaine, ketamine, or both administered via a lumbosacral approach in dogs. American Journal of Veterinary Research, 72(12): 1580-1585, 2011.

El-Khateeb, O.E. Caudal ketamine for relief of pain following anorectal surgery. In: Domino EF. Status of Ketamine In Anesthesiology. Ann Arbor, 1990.

Fantoni, D.T.; Cortopassi, S.R.G.; Bernardi, M.M. Anestésicos Intravenosos e Outros Parenterais. In: Spinosa, H. S.; Górniak, S. L.; Bernardir, M. 
M., Farmacologia aplicada à medicina veterinária. Rio de Janeiro: Guanabara Koogan, 2011. p.129-139.

Farmawy, M.S.; Rashad, M.M. Preemptive analgesia by peritonsillar ketamine versus ropivacaine for post-tonsillectomy pain in children. Egyptian Journal of Anaesthesia, 30(1): 1-5, 2014.

Farnia, M.R.; Jalali, A.; Vahidi, E.; Momeni, M.; Seyedhosseini, J.; Saeedi, M. Comparison of intranasal ketamine versus intravenous morphine in reducing pain in patients with renal colic. American Journal of Emergency Medicine, 35(3): 434-437, 2016.

Fielding, C.L.; Brumbaugh, G.W.; Matthews, N.S.; Peck, K.E.; Roussel, A.J. Pharmacokinetics and clinical effects of a subanesthetic continuous rate infusion of ketamine in awake horses. American Journal of Veterinary Research, 67(9): 1484-1490, 2006.

$\mathrm{Fu}$, E.S.; Miguel, R. Scharf, J.E. Preempitive ketamine decreases post-operatorative narcotic requirements in patients undergoing abdominal surgery. Anesthesia \& Analgesia, 84(5): 1294, 1997.

Gomes De Segura, I.A.; DeRossi, R.; Santos, M.; San-Roman, J.L.; Tendillo, F.J. Epidural injections of ketamine for perineal analgesia in the horse. Veterinary Surgery, 27(4): 217-384, 1998.

Guinto-Enriquez, G.; Esqiquez, R.Y.; Reyedes De Castro, I. Epidural injection of ketamine hydrochloride: $\mathrm{Na}$ experimental study in rats. In: Domino EF, Ed. Status of Ketamine in Anesthesiology, Ann Arbor, 1990.

Hong, B.; Lim, C.S.; Kim, Y.H.; Lee, J.U.; Kim, Y.M.; Jung, C.; Jo, Y. Comparison of topical and non-ketamine ropivacaine in post-surgical pain in children submitted to tonsillectomy: a randomized, double-blind controlled trial. Journal of Anesthesia, 31(4): 559-564, 2017.

Hosseini Jahromi, S.A.; Hosseini Valami, S.M.; Hatamian, S. Comparison between effect of lidocaine, morphine and ketamine spray on post-tonsillectomy pain in children. Anesthesiology and Pain Medicine, 2(1): 1721, 2012.

Kawamata, T.; Omote, K.; Sonoda, H.; Kawamata, M.; Namiki, A. Analgesic mechanisms of ketamine in the presence and absence of peripheral inflammation. Anesthesiology, 93(2): 520-528, 2000.
Khajavi, M.R.; Navardi, M.; Moharari, R.S.; Pourfakhr, P.; Khalili, N.; Etezadi, F.; Imani, F. Combined ketamine-tramadol subcutaneous wound infiltration for multimodal postoperative analgesia: a double-blinded, randomized controlled trial after renal surgery. Anesthesiology and Pain Medicine, 6(5), 2016.

Kitahana, L.M.; Taub, A.; Kosaka, Y. Laminaspecific suppressions of dorsal-horn unit activity by ketamina hydrockloride. Anesthesiology, 38(1):4-11, 1973.

Kock, M.D.; Loix, S.; Lavand'homme, P. Ketamine and peripheral inflammation. CNS Neuroscience \& Therapeutics, 19: 403-410, 2013.

Kwok, R.F.K.; Lim, L.; Chan, M.T.V.; Gin T.; Chiu W.K.Y. Preoperative ketamine improves postoperative analgesia after gynaecologic laparascopic surgery. Anesthesia \& Analgesia, 98(4): 1044-1049, 2004.

Lankveld, D.P.K.; Bull, S.; Dijki, P.V.; FinkGremmels, J.; Hellebrekers L.J. Ketamine inhibits LPS-induced tumour necrosis factoralpha and interleukin-6 in na equine macrophage cell line. Veterinary Research, 36(2): 257-262, 2005.

Lee, I.O.; Kim, W.K.; Kong, M.H.; Lee, M.K.; Kim, N.S.; Choi Y.S.; Lim S.H. No enhancement of sensory and motor blockade by ketamine added to ropivacaineinterscalene brachial plexus blockade. Acta Anaesthesiologica Scandinavica, 46(8): 821826, 2002.

Liao, E.; Mermelsteins, F.; Wright, C.; Carr, D.B. Relative Analgesic Potencies of Intranasal Ketamine and Intranasal Morphine Compared to Intravenous Morphine. American Academy of Pain Medicine, 2008.

Lin, H., Anestésicos Dissociativos. In: Tranquilli, W.J.; Thurmon, J.C.; Grimm, K.A. Anestesiologia e Analgesia Veterinária. São Paulo: Roca, 2013. p. 335-384.

Liu, F.L.; Chen, T.L.; Chen, R.M. Mecanismo de imunossupressão induzida por cetamina. Acta Anaesthesiologica Taiwanica, 50: 172-177, 2012.

Lopez-Sanroman, F.J.; Cruz, J.M.; Santos, M.; Mazzini, R.A.; Tabanera, A.; Tendillo, F.J. Evaluations of the local analgesic effect of ketamine in the palmar digital nerve block at the base of the proximal sesamoid (abaxial 
sesamoid block) in horses. American Journal of Veterinary Research, 64(4): 475-478, 2003.

Luft, A.; Mendes, F.F. S (+) Cetamina em Baixas Doses: Atualização. Revista Brasileira de Anestesiologia, 55(4), 2005.

Luna, S.P.L.; Neto, F.J.T.; Aguiar, A.J.A. Anestesiologia em Pequenos Animais. Botucatu: Fmvz Unesp, 2016.

Massopust, L.C.; Wolin, L.R.; Albin, M.S. The effects of a new phencyclidine derivative and diazepione derivative on the electroencephalographic and behavioral responses in the cat. Tower International Technomedical Journal of Life Sciences, 3(1), 1973.

Marjani, M.; Akbarinejad, V.; Bagheri, M. Comparison of intranasal and intramuscular ketamine midazolam combination in cats, Veterinary Anaesthesia and Analgesia, 42(2): 178-181, 2015.

Moharari R.S.; Valizade, A.; Najafi, A.; Etezadi, F.; Hosseini, S.R.; Khasharayar, P.; Khajavi, M.R.; Mojtahedzadeh, M. Analgesic efficacy of nephrostomy tract infiltration of bupivacaine and ketamine after tubeless percutaneous nephrolithotomy: a prospective randomized trial. Iranian Journal of Pharmaceutical Research, 15(2): 619-626, 2016.

Moharari, R.S.; Najafi, A.; Khajavi, M.R.; Moharari, G.S.; Nikoobakht M.R. Intraurethral instillation of ketamine for male rigid cystoscopy. Journal of Endourology, 24(12): 2033-2036, 2010.

Miyasaka M.; Domino E.F. Neuronal mechanisms of ketamine induced anesthesia. International Journal of Neuropharmacology, 7(6): 557573, 1968.

Motov, S.; Mai, M.; Pushkar, I.; Likourezoz, A.; Drapkin, J.; Yasavolian, M.; Brady, J.; Homel, P.; Fromm, C. Prospective randomized, doubledummy Trial comparing intravenous push dose of low dose ketamine to short infusion of low dose ketamine for treatment of moderate to severe pain in the emergency department. American Journal of Emergency Medicine, 35(8): 1095-1100, 2017.

Muir, W.W. NMDA receptor antagonists and pain: ketamine. Veterinary Clinics of North America: Equine Practice, 26(3): 565-578, 2010.

Ohtani, M.; Kikuchi, H.; Kitahana, L.M.; Taub, A.; Toyooka, H.; Hannaoka, K.; Dohi, S. Effects of ketamine on nociceptive cells in the mediai medullary reticular formations of the cat. Anesthesiology, 51(5): 414-427, 1979.

Othman, A.H.; El-Rahman, A.M.A.; El Sherif, F. Efficacy and safety of ketamine added to local anesthetic in modified pectoral block for management of postoperative pain in patients undergoing modified radical mastectomy. Pain Physician, 19(7): 485-494, 2016.

Parvizrad, R.; Pakniyat, A.; Malekianadeh, B. Comparing the analgesic effect of intranasal with intravenous ketamine in isolated orthopedic trauma: A randomized clinical trial. Turkish Journal of Emergency Medicine, 17(3): 99-103, 2017.

Pederson, J.L.; Galle T.S.; Kehlet, H.M. Peripheral analgesic effects of ketamine in acute inflammatory pain. Anesthesiology, 89(1): 5866, 1998.

Preskorn, S.H. Ketamine: the hopes and obstacles. Biological Psychiatry, 72: 522-523, 2012.

Pourmand, A.; Mazer-Amirshasi, M.; Royall, C.; Alhawas, R.; Shesser, R. Low dose ketamine use in the emergency department, a new direction in pain management. The American Journal of Emergency Medicine, 35(6): 918921, 2017.

Rascón-Martínez, D.M.; Carrillo-Torres, O.; Ramos-Nataren, R.G.; Rendón-Jaramillo, L. Advantages of ketamine as a perioperative analgesic. Revista Médica del Hospital General de México, 79(4), 2016.

Rédua, M.A.; Valvadão, C.A.A.; Duque, J. C.; Balestrero, L.T. The pré-emptive effect of ketamine on wound sensitivity in horses tested by using Von Frey filaments. Veterinary Anaesthesia and Analgesia, 29(4): 200-206, 2002.

Robson, M.J.; Elliot, M.; Seminerio, M. J.; Matsumoto, R.R. Evaluation of sigma receptors $(\sigma)$ on antidepressant effects of ketamine in vitro and in vivo. European Neuropsychopharmacology, 22(4): 308317.2012.

Romagnoli, N.; Bektas, R.N.; Kutter, A.P.; Barbarossa, A.; Roncada, P.; Hartnack, S.; Bettschart-Wolfensberger, R. Pharmacokinetics of ketamine and norketamine enantiomers after racemic or S-ketamine IV bolus administration in dogs during sevoflurane anaesthesia. Research in Veterinary Science, 112: 208213, 2017. 
Rosseland, L.A.; Stubhand, A.; Sandberg, L.; Breivik, H. Intra-articular (IA) catheter administration of postoperative analgesics. A new trial design allows evaluation of baseline pain, demonstrates large variation in need of analgesics, and finds no analgesic effect of IA ketamine compared with IA saline. Pain, 104(1-2): 25-42, 2003.

Sawyer, D.C.; Rech, R.H.; Durham, R.A. Does ketamine provide adequate visceral anlgesia when used alone or in combination with acepromazine, diazepam, or butorphanol in cats. Journal of the American Animal Hospital Association, 29: 257, 1993.

Sawyer, D.C.; Rech, R.H.; Durham, R.A. Efects of ketamine and combinations with acetylpromazine, diazepam, or butorphanol on visceral nocicptionsin the cat.Em: Domino E.F, Ed. Status of ketamine in anesthesiology, Ann Arbor, 1990.

Stoetzer, C.; Martell, C.; De La Roche, J.; Leffler, A. Inhibition of voltage-gated na+ channels by bupivacaine is enhanced by the adjuvants buprenorphine, ketamine, and clonidine. Regional Anesthesia and Pain Medicine, 42(4):462-468, 2017.

Stubburg, A.; Breivik, H.; Eide, P.K.; Kreunen, M.; Foss, A. Mapping of punctuavehyperalgesia around a surgical incision demonstrates that ketamine is a powerful suppressor of central sensitization to pain following surgery. Acta
Anaesthesiologica Scandinavica, 41: 1124, 1997.

Tsao, C.; Wu, C. Modulating effects of ketamine on inflammatory response in sepsis. Acta Anaesthesiologica Taiwanica, 145-146, 2012. TvwrkoY, M.; Oren, M.; Vaskovich, M. Dashkovvsky, I.; Kissin, I. Ketamine enhances local anesthesic and analgesic effects of bupivacaine by-peripheral mechanism: A study in postoperative patients. Neuroscience Letters. 215(1): 5-8, 1996.

Warncke, T.; Jorum, F.; Stubhaug, A. Local treatment with the N-methyl-D-aspartate receptor antagonist, ketamine, inhibits development of secondary hyperalgesia in man by a peripheral actions. Neuroscience Letters, 227(1): 1-4, 1996.

Woolfe, C.J.; Thompson, S.W.N. The inductions and maintenance of central sensitizations is dependent on N-MethylD-Aspartate receptor activations: Implications for the treatament of post-injury pain hypersensibivity state, Pain, 44(3): 293-299, 1991.

Xingwei, X.; Xin, G.; Peng, Z.; Tao, F.; Bowen, D.; Xiaoming, K.; Wu, J.; Ning, L.; Jiesbou, L. Low-dose ketamine pretreatment reduces oxidative damage and inammatory response following $\mathrm{CO} 2$ pneumoperitoneum in rats. Clinical and Investigative Medicine, 37(3), 2014. 